\title{
Definiciones, criterios diagnósticos y valoración de terminalidad en enfermedades crónicas oncológicas y no oncológicas
}

\author{
José Amado* 1,2,a ; Teodoro Oscanoa 1,3,a
}

\section{RESUMEN}

Los cambios demográficos mundiales han producido nuevos retos en el sistema de salud. La atención de pacientes de mayor edad y con problemas de salud más complejos se ha incrementado de manera importante y sobrepasan nuestra capacidad de respuesta; el Perú no escapa a esta realidad. Estos pacientes necesitan cada vez mayores cuidados y la enfermedad crónica en fase avanzada requiere un cambio de enfoque; de los tratamientos agresivos con fines curativos hacia un enfoque paliativo, de mejora de la calidad de vida, asociado a un importante contenido social. La enfermedad en fase terminal se sitúa en la etapa final de la vida, en la que existe daño irreversible y múltiples síntomas somáticos, psicológicos y espirituales, con gran impacto en el paciente y su familia. Se ha estudiado con relativa frecuencia la enfermedad oncológica en fase terminal, sin embargo, las no oncológicas tienen mayor frecuencia y sobrevida. El objetivo de la presente revisión es hacer un compendio de las definiciones operacionales de esta condición, describir criterios diagnósticos específicos de terminalidad, resumir instrumentos para identificar necesidad de cuidados paliativos y escalas de pronóstico publicadas en la literatura científica.

Palabras clave: Enfermedad crónica; Cuidado terminal; Cuidados paliativos; Pronóstico (Fuente: DeCS BIREME).

\section{Definitions, diagnostic criteria and terminality assessment of cancer and non- cancer chronic diseases}

\section{ABSTRACT}

Global demographic changes have generated new challenges in the health system. Treating increasingly older patients with more complex health problems has significantly raised and exceeded our response capacity, and Peru is not indifferent to this situation. These patients need more and more care, and late-stage chronic diseases require an approach change: from aggressive healing treatments to palliative ones which improve quality of life and are associated with an important social content. Late-stage diseases occur in the final stage of life, where irreversible damage; multiple somatic, psychological and spiritual symptoms; as well as great impact in the patients and their families take place. Late-stage cancer diseases have been studied on a relatively frequent basis; however, non-cancer diseases occur more often and result in better survival rates. This review article aims at gathering the operational definitions of this condition, describing the specific diagnostic criteria of terminality, and summarizing the instruments to identify the need of palliative care and the prognostic scales published in the scientific literature.

Keywords: Chronic disease; Terminal care; Palliative care; Prognosis (Source: MeSH NLM).

\footnotetext{
1 Universidad de San Martín de Porres, Facultad de Medicina Humana, Instituto de Investigación. Lima, Perú.

2 Hospital Nacional Edgardo Rebagliati Martins, EsSalud. Lima, Perú.

3 Hospital Guillermo Almenara Irigoyen-EsSalud. Lima, Perú.

a Médico Internista, Doctor en Medicina.

*Autor corresponsal
} 


\section{INTRODUCCIÓN}

La mayor esperanza de vida, el avance científicotecnológico y los cambios socioculturales han producido un incremento significativo de enfermedades crónicas degenerativas y neoplásicas, muchas personas viven más, pero con mayores comorbilidades. El envejecimiento de la población representa un reto importante para los servicios de salud en el mundo. Muchos de estos enfermos, al final de su vida, padecen un sufrimiento intenso y precisan una atención sanitaria y social que implica a todos los ámbitos asistenciales ${ }^{(1,2)}$.

En Europa se estima que más de la mitad de personas que fallecen han pasado un difícil proceso de deterioro en el último año de su vida. Se estima que entre $8 \%$ y $22 \%$ de las hospitalizaciones pueden corresponder a enfermos en este periodo. En Latinoamérica y nuestro país esta realidad es muy frecuente y es poco lo que se ha avanzado en este contexto ${ }^{(1,3-7)}$.

La tendencia mundial en salud es la atención centrada en la persona, de calidad y a costes razonables, que permita una vida digna hasta el momento de la muerte; con la posibilidad de escoger donde pasar este último momento, por ejemplo, rodeado de la familia, lo que significa el retorno al domicilio como lugar de fallecimiento, en lugar del hospital. Esta realidad revela la necesidad de replantearnos los objetivos de la medicina actual que, hasta ahora, se ha centrado de manera excesiva en un enfoque curativo. Callahan, desde el año 2000, abogaba por reconocer a la muerte natural en paz como un objetivo del mismo valor e importancia que la lucha contra las enfermedades y la prolongación de la vida ${ }^{(8-10)}$.

La realización de estudios relacionados a las fases finales de enfermedad crónica, oncológica y no oncológica, requiere definiciones operacionales claras (criterios de terminalidad), por lo que es de suma importancia conocer instrumentos o escalas para determinar necesidades de cuidado y valoración pronóstica, los cuales a su vez servirán para una intervención adecuada e informada, evitar medidas de dudosa eficacia y disminuir los costos relacionados (económicos y sociales) ${ }^{(9,11,12)}$.

\section{Estrategia de búsqueda}

Para la presente revisión se realizó búsqueda en bases de datos bibliográficas como Pubmed, CINAHL, SciELO, LILACS y Google académico, entre estudios primarios, revisiones, consensos y guías clínicas, desde 1998 hasta noviembre de 2018, en idioma inglés y español. Se utilizó como términos de búsqueda: enfermedad crónica avanzada, cáncer avanzado, enfermedad terminal, estado final de vida, cuidados terminales, etapa final de enfermedad, definiciones, criterios, instrumento de valoración y escala de pronóstico.

\section{Enfermedad en fase terminal}

Identificar al paciente con enfermedad en fase terminal es un problema para el personal de salud. Muchos de estos enfermos, al final de su vida, padecen intenso sufrimiento y precisan atención sanitaria y social que implica a todos los ámbitos asistenciales; pero a la vez el paciente y la familia tienen miedo de ser abandonados o discriminados ${ }^{(2,13)}$.

La frase "paciente terminal" estigmatiza a la persona y no debería utilizarse, sin embargo, la evolución de las enfermedades crónicas (oncológicas y no oncológicas) produce situaciones complejas en quienes las padecen, y es cuando términos como fase terminal y situación de últimos días son necesarios. Más que como una clasificación, estos términos son útiles para identificar las necesidades $y$ cuidados especiales que ayuden al paciente y a su familia. Varias publicaciones se refieren a esta etapa del paciente como final de la vida (end of life).

La tabla 1 presenta definiciones operacionales relacionadas a enfermedad en fase terminal. Destacan los consensos de la Organización Mundial de la Salud y otras entidades internacionales como la International Association for Hospice \& Palliative Care (IAHPC), Sociedad Española de cuidados paliativos (SECPAL) y la Asociación Latinoamericana de Cuidados Paliativos (ALCP) que buscan poner en agenda este tema en las distintas regiones del mundo para mejorar la atención integral y humanizada ${ }^{(1,3,9,13,14)}$.

Tabla 1. Definiciones operacionales relacionadas a enfermedad en fase terminal

\section{Enfermedad en fase terminal}

Etapa final de una enfermedad avanzada y progresiva, donde existe daño irreversible y no hay tratamiento curativo posible. Múltiples e intensos síntomas, cambiantes y multifactoriales. Pérdida de la autonomía o fragilidad progresiva. Pronóstico de vida limitado, gran impacto emocional en el paciente, la familia y el equipo terapéutico. Asociada a alta demanda y uso de recursos $(17,19,26,27)$.

\section{Enfermedad oncológica en fase terminal}

Cáncer con diagnóstico histológico demostrado en estadio clínico IV, metástasis cerebral, medular, hepática o pulmonar múltiple; que ha recibido terapéutica estándar eficaz y/o se encuentra en situación de escasa o nula posibilidad de respuesta al tratamiento activo específico ${ }^{(13)}$. 


\section{Enfermedad no oncológica en fase terminal}

Enfermedad o insuficiencia crónica de órgano no reversibles, independientemente de la causa primigenia que las originó, en el estadio más avanzado y sin respuesta al mejor tratamiento disponible ${ }^{(13)}$.

\section{Situación de últimos días o agonía}

Periodo final del ciclo vital de las personas con marcado declinar funcional, alteración de signos vitales, previsión de muerte en horas o días e irreversibilidad de la situación. Es uno de los momentos más delicados de la enfermedad del paciente ${ }^{(13,26)}$.

Las causas o enfermedades que pueden llevar al paciente a esta condición son tan diversas como la medicina misma; además, por la evolución epidemiológica y por el avance de la ciencia, con frecuencia son más de una. En la tabla 2 se resume los criterios específicos por etiología, propuestos en la literatura revisada. Estos criterios son numerosos y comprenden a muchas especialidades médicas, pero el principal objetivo sería detectar al paciente que está en la fase avanzada y derivarlo para un cuidado más adecuado para sus necesidades, ya existiendo en el mundo la especialidad y la unidad de cuidados paliativos.

Tabla 2. Criterios diagnóstico específicos de enfermedad crónica en fase terminal, oncológica y no oncológica

Criterios de terminalidad en el paciente oncológico: $(2,7,13,28,29)$

Presencia de una enfermedad oncológica avanzada, progresiva e incurable; con diagnóstico histológico demostrado (estadio clínico IV).

Metástasis cerebral, medular, hepática o pulmonar múltiple.

Haber recibido terapéutica estándar eficaz y/o encontrarse en situación de escasa o nula posibilidad de respuesta al tratamiento activo específico para su patología oncológica.

Criterios de terminalidad en el paciente no oncológico: ${ }^{(5,6,11-13,21,30)}$

Insuficiencia cardiaca con disnea basal $\geq$ III de la NYHA (New York Health Association) o fracción de eyección del ventrículo izquierdo $<20 \%$ o persistencia de síntomas a pesar de tratamiento óptimo. NO CANDIDATOS trasplante de órgano.

Insuficiencia respiratoria crónica con disnea basal $\geq$ III de la MRC (Medical Research Council) y/o saturación de oxígeno basal $<90 \%$ y/o oxigenoterapia domiciliaria continua. NO CANDIDATOS trasplante de órgano.

Insuficiencia renal crónica en estadio 5 según NKF (National Kidney Foundation), tasa de filtrado glomerular $<15 \mathrm{ml} / \mathrm{min}$ según la fórmula de Crockoft-Gault, o creatina sérica basal $\geq 3 \mathrm{mg} / \mathrm{dl}$ en pacientes NO CANDIDATOS a tratamiento sustitutivo (diálisis) o trasplante.

Hepatopatía crónica con datos clínicos, analíticos y/o ecográficos de hipertensión portal y/o insuficiencia hepatocelular grave : estadio C (según escala Child-Pugh) o MELD $>30$ (Model for End-stage Liver Disease). NO CANDIDATOS trasplante de órgano.

Demencia en estadio 7 según las escalas GDS (Global Deterioration Scale de Reisberg) y FAST (Functional Assesment Staging).

Enfermedad neurológica crónica avanzada (enfermedad cerebrovascular, esclerosis lateral amiotrófica, esclerosis múltiple o enfermedad de Parkinson) con deterioro cognitivo establecido (Escala de Pfeiffer con más de 7 errores) y/o deterioro funcional para las ABVD (Actividades básicas de la vida diaria) establecido (Dependencia total según test de Katz). 
Enfermedad de Parkinson: la clasificación de Hoen y Yahr en el estadio 5.

Infección por virus de la inmunodeficiencia humana en estadio SIDA (síndrome de inmunodeficiencia adquirida) refractaria a tratamiento antirretroviral de gran actividad.

Tuberculosis extensamente resistente (resistente a isoniacida, rifampicina, una fluorquinolona y un inyectable de segunda línea) con evolución desfavorable

\section{Otros criterios: ${ }^{(13,31)}$}

Dependencia funcional total persistente: Índice de Katz con 6 ítems positivos de dependencia para ABVD.

Deterioro cognitivo importante persistente: 8-10 errores en el cuestionario mental de Pfeiffer. Úlceras por decúbito persistentes (estadio III-IV).

Con la finalidad de hacer más objetiva la detección de la fase final de la enfermedad se han creado instrumentos para identificar esta situación (tabla 3), pero la tendencia actual es más que detectar la enfermedad, implica también detectar la necesidad de cuidados del paciente. Para nuestro medio, la Escala de Necesidad Paliativa
(NECPAL) de la Sociedad Española de Cuidados Paliativos es la más entendible y útil, sobre todo para el nivel de atención primario; sin embargo, cabe mencionar que la The Supportive \& Palliative Care Indicators Tool (SPICT-TM) también tiene una versión en español que es fácil de aplicar.

Tabla 3. Instrumentos para identificar enfermedad en fase terminal y necesidad de cuidados paliativos

\begin{tabular}{|c|c|c|c|c|c|c|}
\hline Instrumento & País & Año & Criterios & $\begin{array}{l}\text { Idioma } \\
\text { publicación }\end{array}$ & Organismo & Referencias \\
\hline $\begin{array}{l}\text { Hospice } \\
\text { Care } \\
\text { and } \\
\text { Palliative } \\
\text { Care }\end{array}$ & EE. UU.* & $\begin{array}{l}1996 \\
\text { Última } \\
\text { versión } \\
2018\end{array}$ & $\begin{array}{l}\text { - Clínico } \\
\text { - Asistencial } \\
\text { - Preferencias } \\
\text { del paciente } \\
\text { - Criterios específicos }\end{array}$ & Inglés & $\begin{array}{l}\text { National } \\
\text { Hospice and } \\
\text { Palliative Care } \\
\text { Organization } \\
\text { (NHPCO) }\end{array}$ & $(19,20)$ \\
\hline $\begin{array}{l}\text { NECPAL } \\
\text { CCOMS-ICOC } \\
3.1\end{array}$ & España & $\begin{array}{l}2010 \\
\text { última } \\
\text { versión } \\
2017\end{array}$ & $\begin{array}{l}\text { - Enfermedad } \\
\text { avanzada } \\
\text { - Síntomas } \\
\text { intensos }\end{array}$ & Español & $\begin{array}{l}\text { Sociedad española } \\
\text { de cuidados } \\
\text { paliativos } \\
\text { (SECPAL) }\end{array}$ & $(13,24,32)$ \\
\hline $\begin{array}{l}\text { Prognostic } \\
\text { Indicator } \\
\text { Guidance } \\
\text { (PIG). Gold } \\
\text { Standards } \\
\text { Framework }\end{array}$ & Reino Unido & $\begin{array}{l}2011 \\
\text { última } \\
\text { versión } \\
2016\end{array}$ & $\begin{array}{l}\text { - Impacto emocional } \\
\text { - Pronostico de vida } \\
\text { - Impacto emocional } \\
\text { - necesidades de cuidado } \\
\text { - Indicadores clínicos } \\
\text { específicos }\end{array}$ & Inglés & $\begin{array}{l}\text { Royal College } \\
\text { of General } \\
\text { Practitioners }\end{array}$ & $(25,26)$ \\
\hline $\begin{array}{l}\text { The Supportive } \\
\& \text { Palliative } \\
\text { Care Indicators } \\
\text { Tool } \\
\text { (SPICT-TM) }\end{array}$ & Escocia & $\begin{array}{l}2015 \\
\text { última } \\
\text { versión } \\
2018\end{array}$ & $\begin{array}{l}\text { - Deterioro de salud } \\
\text { - Condiciones límites } \\
\text { de vida } \\
\text { - Tratamiento y planes }\end{array}$ & $\begin{array}{l}\text { Inglés, } \\
\text { español } \\
\text { y otros } \\
7 \text { idiomas }\end{array}$ & $\begin{array}{l}\text { University of } \\
\text { Edinburgh }\end{array}$ & (27) \\
\hline
\end{tabular}




\begin{tabular}{|c|c|c|c|c|c|c|}
\hline Instrumento & País & Año & Criterios & $\begin{array}{l}\text { Idioma } \\
\text { publicación }\end{array}$ & Organismo & Referencias \\
\hline $\begin{array}{l}\text { Australian } \\
\text { Commission } \\
\text { on Safety and } \\
\text { Quality in } \\
\text { Health Care } \\
\text { (ACSQHC) }\end{array}$ & Australia & 2015 & $\begin{array}{l}\text { - Deterioro de salud } \\
\text { - Condiciones límites } \\
\text { de vida }\end{array}$ & Inglés & $\begin{array}{l}\text { Australian } \\
\text { Commission } \\
\text { on Safety } \\
\text { and Quality } \\
\text { in Health } \\
\text { Care (ACSQHC) }\end{array}$ & $(17,28)$ \\
\hline
\end{tabular}

*EE. UU. : Estados Unidos de Norteamérica

Actualmente, se prefiere utilizar el término "enfermedad avanzada" en vez de "enfermedad terminal", y se habla de cuidados paliativos como una filosofía de vida, en la que un equipo humano multidisciplinario (conformado por médico, enfermera, psicóloga, trabajadora social, terapistas, guía espiritual y otros) brinda acompañamiento y soporte a una persona y su familia que lo necesita, sin adelantar ni retrasar el proceso natural de su muerte. No solamente se lucha contra una enfermedad, sino que se tratan todos los padecimientos del paciente ${ }^{(3,9,14,15)}$.

Los cuidados paliativos en enfermedades oncológicas avanzadas han tenido logros importantes en mejorar la calidad de vida de estos pacientes. Sin embargo, los fallecimientos por enfermedades no oncológicas (insuficiencias orgánicas y enfermedades neurodegenerativas) son más numerosos; y las necesidades de estos pacientes y sus familias son similares, incluso más prolongadas y reciben peores cuidados. De acuerdo al principio de justicia, es una obligación ética potenciar esta atención ${ }^{(1,12,16)}$.

Cabe mencionar que existe enfermedad no recuperable en cuadros agudos, relacionada a evolución de infecciones sistémicas con falla multiorgánica, hemorragias cerebrales masivas o consecuencias de eventos traumáticos graves, como se reporta en Reino Unido y Australia. Estos casos tienen sus propios criterios y requieren un enfoque especial en los servicios de emergencias o cuidados críticos, pero no serán considerados en esta revisión ${ }^{(17,18)}$.

\section{Escalas e instrumentos de valoración en enfermedad en fase terminal}

La medicina científica es, fundamentalmente, una ciencia probabilística, pero dar pronósticos exactos en esta etapa final de la vida podría ser un error. Sin embargo, conocer la magnitud del problema de salud y determinar un pronóstico del paciente es fundamental para plantear la toma de decisiones al final de la vida.

Esta es una de las mayores dificultades a las que se va a enfrentar el profesional de salud (12,19,20). Comunicar a la familia o al paciente la situación de salud es indispensable, pero los médicos no fuimos preparados adecuadamente en la formación profesional de pregrado, e incluso, de posgrado para dar malas noticias. El momento más adecuado para hablar de esto es cuando el paciente se encuentra estable clínicamente.

En muchos países se puede establecer voluntades anticipadas y planes avanzados para el final de la vida; en Perú, la ley todavía no lo contempla. Informar sobre fechas o plazos exactos en la mayoría de veces es un error, sin embargo podemos dar tiempos aproximados de sobrevida (entre semanas-meses, días-semanas u horas-días) lo cual es muy importante para preparar a la familia y enfrentar el evento final de la vida (la muerte) que ocurrirá de todos modos ${ }^{(11,12,21)}$.

La evolución de la enfermedad crónica es variable. En el cáncer el deterioro es lento y progresivo, y, la mayoría de veces, finaliza por cuadros infecciosos agudos en menos de seis meses de diagnosticada la fase terminal. La enfermedad no oncológica es más lenta, con varios episodios de descompensación aguda que mejoran y vuelve al estado basal. Esto también varía según el tipo o etiología de la enfermedad y los cuidados que reciba el paciente ${ }^{(11,21,22)}$.

Actualmente, existen múltiples herramientas diagnósticas y de pronóstico disponibles (http://eprognosis.ucsf.edu), que se crearon inicialmente para pacientes con cáncer, pero que, posteriormente, fueron adaptadas también para las enfermedades no oncológicas (Tabla 4).

Las escalas más antiguas y conocidas son la de Karnofski y el índice de Zubrod o performance status ECOG (Eastern Cooperative Oncology Group). Sin embargo, en los últimos años la más utilizada es la escala funcional paliativa (Palliative Performance Scale, PPS) que ha acumulado mayor evidencia y cuyo empleo se recomienda ${ }^{(23-25)}$. 
Tabla 4. Escalas pronósticas para pacientes con enfermedad en fase terminal oncológica y no oncológica

\begin{tabular}{|c|c|c|c|c|c|c|}
\hline Escala & País & Año & Criterios & $\begin{array}{l}\text { Tipo de } \\
\text { pacientes }\end{array}$ & Interpretación & Referencias \\
\hline $\begin{array}{l}\text { Escala } \\
\text { pronóstica } \\
\text { de Karnofski }\end{array}$ & $\begin{array}{l}\text { EE. UU.* } \\
\text { Australia }\end{array}$ & $\begin{array}{l}1948 \\
2011\end{array}$ & $\begin{array}{l}\text { Índice } \\
\text { funcional } \\
\text { Subjetivo }\end{array}$ & Oncológicos & $\begin{array}{l}\text { Menor puntuación, } \\
\text { peor pronóstico }\end{array}$ & $(28)$ \\
\hline $\begin{array}{l}\text { Escala de } \\
\text { Zubrod o } \\
\text { performance } \\
\text { status ECOG } \\
\text { (Eastern } \\
\text { Cooperative } \\
\text { Oncology } \\
\text { Group) }\end{array}$ & EE. UU. & 1982 & $\begin{array}{l}\text { Calidad } \\
\text { de vida }\end{array}$ & Oncológicos & $\begin{array}{l}\text { Validado por } \\
\mathrm{OMS}^{* *}\end{array}$ & (32) \\
\hline $\begin{array}{l}\text { Escala Funcional } \\
\text { Paliativa } \\
\text { (Palliative } \\
\text { Performance } \\
\text { Scale, PPS) }\end{array}$ & $\begin{array}{l}\text { Canadá } \\
\text { versión } 2\end{array}$ & $\begin{array}{l}1996 \\
2001\end{array}$ & $\begin{array}{l}\text { Deambulación, } \\
\text { actividad, } \\
\text { autocuidado, } \\
\text { ingesta, nivel } \\
\text { de conciencia }\end{array}$ & $\begin{array}{l}\text { Oncológicos, } \\
\text { no oncológicos, } \\
\text { paliativos agudos }\end{array}$ & $\begin{array}{l}\text { Sobrevida a } \\
6 \text { meses }\end{array}$ & $(29,30)$ \\
\hline $\begin{array}{l}\text { PaP Score } \\
\text { (Palliative } \\
\text { Prognostic Score) } \\
\text { D-PaP Score }\end{array}$ & Italia & $\begin{array}{l}1998 \\
2011\end{array}$ & $\begin{array}{l}\text { Síntomas, } \\
\text { Karnoski, } \\
\text { laboratorio, } \\
\text { severidad, } \\
\text { agrega delirio }\end{array}$ & Oncológicos & $\begin{array}{l}\text { Sobrevida a } \\
30 \text { días }\end{array}$ & (33) \\
\hline $\begin{array}{l}\text { PPI (Palliative } \\
\text { Prognostic Index) }\end{array}$ & Japón & 1999 & $\begin{array}{l}\text { PPS, edema, } \\
\text { disnea, delirio, } \\
\text { ingesta }\end{array}$ & $\begin{array}{l}\text { Oncológicos y } \\
\text { no oncológicos }\end{array}$ & $\begin{array}{l}\text { Sobrevida a } \\
3 \text { semanas }\end{array}$ & (21) \\
\hline $\begin{array}{l}\text { Chuang } \\
\text { Predictor Score }\end{array}$ & Taiwan & 2004 & $\begin{array}{l}\text { Metástasis, } \\
\text { severidad de } \\
\text { signos y síntomas }\end{array}$ & Oncológicos & $\begin{array}{l}\text { Sobrevida a } \\
2 \text { semanas }\end{array}$ & $(23,34)$ \\
\hline $\begin{array}{l}\text { PiPs (Prognosis } \\
\text { in Palliative } \\
\text { Care Study) }\end{array}$ & $\begin{array}{l}\text { Reino } \\
\text { Unido } \\
\text { Versión } 2\end{array}$ & $\begin{array}{l}2011 \\
2018\end{array}$ & $\begin{array}{l}\text { Diagnóstico, } \\
\text { metástasis, síntomas, } \\
\text { valoración mental, } \\
\text { funcional y general }\end{array}$ & Oncológicos & $\begin{array}{l}\text { Sobrevida días, } \\
\text { semanas, meses }\end{array}$ & $(35,36)$ \\
\hline $\begin{array}{l}\text { FAST (Functional } \\
\text { Assessment } \\
\text { Staging Test) }\end{array}$ & EE. UU. & 1982 & $\begin{array}{l}\text { Condición general, } \\
\text { deterioro funcional, } \\
\text { expectativa de vida }\end{array}$ & Demencia & $\begin{array}{l}\text { Pronóstico a } \\
6 \text { meses }\end{array}$ & (37) \\
\hline $\begin{array}{l}\text { Índice BODE } \\
\text { (Body mass } \\
\text { index, Degree } \\
\text { of obstruction, } \\
\text { Dyspnoea and } \\
\text { capacity) }\end{array}$ & $\begin{array}{l}\text { EE. UU., } \\
\text { España, } \\
\text { Venezuela }\end{array}$ & 2004 & $\begin{array}{l}\text { IMC, FEV1, } \\
\text { disnea, test } \\
\text { marcha }\end{array}$ & $\mathrm{EPOC}^{* * *}$ & $\begin{array}{l}\text { Sobrevida a } \\
4 \text { años }\end{array}$ & $(38,39)$ \\
\hline
\end{tabular}




\begin{tabular}{|c|c|c|c|c|c|c|}
\hline Escala & País & Año & Criterios & $\begin{array}{l}\text { Tipo de } \\
\text { pacientes }\end{array}$ & Interpretación & Referencias \\
\hline Índice PALIAR & España & 2010 & $\begin{array}{l}\text { Edad, } \\
\text { característica } \\
\text { clínicas, } \\
\text { analíticas, } \\
\text { ECOG }\end{array}$ & No oncológicos & $\begin{array}{l}\text { Mortalidad a } \\
6 \text { meses }\end{array}$ & $(21,40)$ \\
\hline
\end{tabular}

*EE. UU.: Estados Unidos de Norteamérica, ** OMS: Organización Mundial de la Salud, ${ }^{* * * E P O C: ~ e n f e r m e d a d ~ p u l m o n a r ~ o b s t r u c t i v a ~ c r o ́ n i c a ~}$

Finalmente, podemos concluir que la enfermedad crónica en fase terminal se presenta en un número importante de personas, asociada a síntomas multifactoriales que involucran a la familia y la sociedad. Las enfermedades de causa no oncológica en este estado son más frecuentes y de mayor duración que las oncológicas, y están relacionadas, muchas veces, a más de una causa. En la literatura científica existen diversas definiciones y se investiga mucho sobre instrumentos de detección y escalas de pronóstico, con el objetivo de mejorar la calidad de atención y de vida del paciente y su entorno, por lo que es necesario que el sistema de salud se adapte a estos importantes cambios.

Contribuciones de autoría: JA y TO han participado en la concepción y el diseño del artículo, análisis e interpretación de datos, revisión crítica del artículo y aprobación de la versión final.

Fuentes de financiamiento: Este artículo ha sido financiado por los autores.

Conflictos de interés: Los autores declaramos no tener ningún conflicto de intereses.

\section{REFERENCIAS BIBLIOGRÁFICAS}

1. Connor SR, Sepulveda Bermedo MC. Global atlas of palliative care at the end of life. London: Worldwide Hospice Palliative Care Alliance. 2014. Disponible en: https://www.who.int/nmh/ Global_Atlas_of_Palliative_Care.pdf

2. Salas Campo T, Vicente Sánchez F, Valentín Maganto V, de Miguel Sánchez C, Sanz Llorente B, Lacasta Reverte A, et al. Guía de cuidados paliativos de la comunidad de Madrid. Madrid. 2008. Disponible en: https://www.bioeticaweb.com/wp-content/ uploads/2014/07/guia_paliativos.pdf

3. Pastrana T, De Lima L, Wenk R, Eisenchlos J, Monti C, Rocafort $\mathrm{J}$, et al. Atlas de cuidados paliativos en Latinoamérica [libro electrónico]. Houston: IAHPC Press. 2012. Disponible en: https:// cuidadospaliativos.org/uploads/2012/10/atlas/Atlas\%20de $\% 20$ Cuidados\%20Paliativos\%20en\%20Latinoamerica.pdf.

4. Amado JP, Vasquez R, Huari RW, Sucari A, Oscanoa T. Patients with end-stage oncologic and non-oncologic disease in emergency service of an Urban Tertiary Hospital. Indian J Palliat Care. 2018; 24(1): 25-7.

5. Le Conte P, Riochet D, Batard E, Volteau C, Giraudeau B, Arnaudet I, et al. Death in emergency departments: a multicenter cross- sectional survey with analysis of withholding and withdrawing life support. Intensive Care Med. 2010; 36(5): 765-72.

6. Rosenwax LK, McNamara BA, Murray K, Mccabe RJ, Aoun SM, Currow DC. Hospital and emergency department use in the last year of life: a baseline for future modifications to end-of-life care. Med J Aust. 2011; 194(11): 570-3.

7. Henson LA, Gao W, Higginson IJ, Smith M, Davies JM, Ellis-Smith C, et al. Emergency department attendance by patients with cancer in their last month of life: a systematic review and meta-analysis. J Clin Oncol. 2015; 33(4): 370-6.

8. Callahan D. Death and the research imperative. N Engl J Med. 2000; 342(9): 654-6.

9. Alonso JP. Cuidados paliativos: entre la humanización y la medicalización del final de la vida. Cien Saúde Coletiva. 2013; 18(9): 2541-8.

10. Navarro Sanz R, Valls Roig M, Castellano Vela E. Atención a pacientes crónicos avanzados no oncológicos con necesidad de cuidados al final de la vida en un hospital de media y larga estancia. Medicina Paliativa. 2011; 18(2): 54-62.

11. Sánchez Isac M. Factores pronósticos de terminalidad no oncológica en cuidados paliativos [Tesis]. Madrid: Universidad Complutense de Madrid. Facultad de Medicina; 2013.

12. Babarro AA, Cano LR, Aguilar AG. Criterios de selección de pacientes con enfermedades no oncológicas en programas $\mathrm{y} / 0$ servicios de cuidados paliativos. Medicina Paliativa. 2010; 17(3): 161-71.

13. Sociedad Española de Cuidados Paliativos (SECPAL). Guía de cuidados paliativos [internet]. 2014. Disponible en: https:// cmvinalo.webs.ull.es/docencia/Posgrado/8-CANCER Y CUIDADOSPALIATIVOS/guiacp.pdf

14. McEwan A, Silverberg JZ. Palliative care in the emergency department. Emerg Med Clin North Am. 2016; 34(3): 667-85.

15. Walsh RI, Mitchell G, Francis L, van Driel ML. What diagnostic tools exist for the early identification of palliative care patients in general practice? A systematic review. J Palliat Care. 2015; 31(2): 118-23.

16. Iglesias ML, Lafuente A. Asistencia al paciente agónico que va a fallecer en urgencias. Anales Sis San Navarra. 2010; 33(Suppl.1): 173-91.

17. Australasian College for Emergency Medicine. Policy on end of life and palliative care in the emergency department (P455). Emerg Med Australas. 2016; 28(5): 617-21.

18. Rosenberg JP, Bullen T, Maher K. Supporting family caregivers with palliative symptom management: a qualitative analysis of the provision of an emergency medication kit in the home setting. Am J Hosp Palliat Med. 2015; 32(5): 484-9.

19. Richards CT, Gisondi MA, Chang CH, Courtney DM, Engel KG, Emanuel $\mathrm{L}$, et al. Palliative care symptom assessment for patients with cancer in the emergency department: validation of the screen for palliative and end-of-life care needs in the emergency 
department instrument. J Palliat Med. 2011; 14(6): 757-64.

20. Bernabeu-Wittel M, Murcia-Zaragoza J, Hernández-Quiles C, Escolano-Fernández B, Jarava-Rol G, Oliver M, et al. Development of a six-month prognostic index in patients with advanced chronic medical conditions: the PALIAR score. J Pain Symptom Manage. 2014; 47(3): 551-65.

21. Ouchi K, Wu M, Medairos R, Grudzen CR, Balsells H, Marcus D, et al. Initiating palliative care consults for advanced dementia patients in the emergency department. J Palliat Med. 2014; 17(3): 346-50.

22. Metola Gómez A, García Romo E, Bellido de Vega S, Carretero Lanchas Y, Azcoitia Álvarez B, Guerrero L. Análisis de la evolución de pacientes en probable situación de últimos días en un servicio de urgencias. Medicina Paliativa. 2013; 20(1): 10-8.

23. Kao YH, Chiang JK. Effect of hospice care on quality indicators of end-of-life care among patients with liver cancer: a national longitudinal population-based study in Taiwan 2000-2011. BMC Palliat Care. 2015; 14: 39.

24. Gómez-Batiste X, Martínez-Muñoz M, Blay C, Amblàs J, Vila L, Costa X, et al. Identifying patients with chronic conditions in need of palliative care in the general population: development of the NECPAL tool and preliminary prevalence rates in Catalonia. BMJ Support Palliat Care. 2013; 3(3): 300-8.

25. Thomas K. Prognostic Indicator Guidance (PIG): the gold standards framework centre in end of life care cic [internet]. 4th edition. London. 2011. Disponible en: https://www. goldstandardsframework.org.uk/cd-content/uploads/files/ General Files/Prognostic Indicator Guidance October 2011.pdf

26. Thomas K, Wilson JA, GSF Team. Prognostic indicator guidance (PIG), national gold standards framework centre in end of life care [Internet]. 6th Edition. 2016. Disponible en: http://www. goldstandardsframework.org.uk

27. Fachado AA, Martínez NS, Roselló MM, Ria JJV, Oliver EB, García RG, et al. Spanish adaptation and validation of the supportive \& palliative care indicators tool - SPICT-ESTM. Rev Saúde Pública. 2018; 52: 3.

28. Australian Commission on Safety and Quality in Health Care. National consensus statement: essential elements for safe and high-quality end-of-life care. Sydney: ACSQHC. 2015. Disponible en: https://www.safetyandquality.gov.au/wp-content/ uploads/2015/05/National-Consensus-Statement-EssentialElements-forsafe-high-quality-end-of-life-care.pdf

29. Vila Arias JM, Pereira Santelesforo S, Lopez Alvarez E, Mendez Muñoz M, Guzmán Gutierrez J, Sanmartín Moreira J. Utilidad del palliative performance scale v2 para la estimación de supervivencia en enfermos con cáncer avanzado. Medicina Paliativa. 2012; 19(4): 133-8.

30. Ho F, Lau F, Downing MG, Lesperance M. A reliability and validity study of the palliative performance scale. BMC Palliat Care. 2008; 7: 10.

31. Forcano García M, Quilez Salas N, Vial Escolano R, Solsona Fernández S, González García P. Predicción de supervivencia en el paciente con cáncer avanzado. Medicina Paliativa. 2015; 22(3): 106-16.

32. Scarpi E, Maltoni M, Miceli R, Mariani L, Caraceni A, Amadori D, et al. Survival prediction for terminally IIl cancer patients: revision of the palliative prognostic score with incorporation of delirium. Oncologist. 2011; 16(12): 1793-9.

33. Nieto Martín MD, Bernabeu Wittel M, de la Higuera Vila L, Mora Rufete A, Barón Franco B, Ollero Baturone M, et al. Adaptation of the palliative prognostic index in patients with advanced medical conditions. Rev Clin Esp. 2013; 213(7): 323-9.
34. Chuang RB, Hu WY, Chiu TY, Chen CY. Prediction of survival in terminal cancer patients in Taiwan: constructing a prognostic scale. J Pain Symptom Manage. 2004; 28(2): 115-22.

35. Gwilliam B, Keeley V, Todd C, Gittins M, Roberts C, Kelly L, et al. Development of Prognosis in Palliative Care Study (PiPS) predictor models to improve prognostication in advanced cancer: prospective cohort study. BMJ. 2011; 343: d4920.

36. Kalpakidou AK, Todd C, Keeley V, Griffiths J, Spencer K, Vickerstaff V, et al. The Prognosis in Palliative Care Study II (PiPS2): study protocol for a multi-centre, prospective, observational, cohort study. BMC Palliat Care. 2018; 17(1): 101.

37. Na HR, Kim SY, Chang YH, Park MH, Cho ST, Han IW, et al. Functional assessment staging (FAST) in korean patients with Alzheimer's disease. J Alzheimers Dis. 2010; 22(1): 151-8.

38. Powrie $D$. The BODE index: a new grading system in COPD. Thorax. 2004; 59(5): 427.

39. Celli BR, Cote CG, Marin JM, Casanova C, Montes de Oca M, Mendez RA, et al. The body-mass index, airflow obstruction, dyspnea, and exercise capacity index in chronic obstructive pulmonary disease. N Engl J Med. 2004; 350(10): 1005-12.

40. Hernández Quiles C, Bernabeu Wittel M, Praena Segovia J, Ternero Vega J, Díez Manglano J, Jarava Rol G, et al. Comparación de la pregunta sorpresa frente a un índice objetivo (Índice PALIAR) para la identificación de situación terminal en pacientes con enfermedades crónicas avanzadas. Rev Clin Esp. 2017; 217(2): 123-4.

\section{Correspondencia:}

José Percy Amado Tineo

Dirección: Jr. Belisario Flores 238 Dpto 301 - Lince. Lima, Perú.

Teléfono: +51990452547

Correo electrónico: jamadot@usmp.pe; jpamadot@gmail.com

Recibido: 03 de noviembre de 2018 Evaluado: 21 de marzo de 2019 Aprobado: 03 de mayo de 2019

(c) La revista. Publicado por Universidad de San Martín de Porres, Perú. (c) BY Licencia de Creative Commons Artículo en acceso abierto bajo términos de Licencia Creative Commons Atribución 4.0 Internacional. (http://creativecommons.org/licenses/by/4.0/)

ORCID iDs

José Amado

Teodoro Oscanoa
(1) https: / / orcid.org/0000-0002-3286-4650

- https: / / orcid.org/0000-0001-9379-4767 